\title{
Re-examination of strains formerly assigned to Hyphopichia burtonii, the phylogeny of the genus Hyphopichia, and the description of Hyphopichia pseudoburtonii sp. nov.
}

\author{
Marizeth Groenewald and Maudy Th. Smith \\ CBS-KNAW Fungal Biodiversity Centre, Uppsalalaan 8, 3584 CT Utrecht, The Netherlands
}

Correspondence

Marizeth Groenewald

m.groenewald@cbs.knaw.nl

\begin{abstract}
On the basis of the nucleotide divergence in the internal transcribed spacer (ITS) domain of the rRNA gene and the D1/D2 domain of the 26S rRNA gene, species of the genus Hyphopichia can be divided into different phylogenetic groups. In this study, three distinct phylogenetic clusters as well as a novel species, Hyphopichia pseudoburtonii sp. nov. with type strain CBS $2455^{\top}(=\mathrm{JCM}$ $\left.16346^{\top}\right)$, are introduced. Mating studies indicate that strains belonging to Hyphopichia pseudoburtonii are heterothallic.
\end{abstract}

The genus Hyphopichia was introduced by Arx \& van der Walt (1976) to accommodate the mycelial yeast species, Pichia burtonii. This heterothallic species is characterized by formation of asci from conjugating yeast cells and by formation of septate hyphae and conidia borne on denticles. Arx \& van der Walt (1976) excluded P. burtonii from the genus Pichia by the latter features and from the genus Saccharomycopsis by the former character. Kurtzman \& Fell (1998) did not accept the reclassification of $P$. burtonii as various other yeasts and other dimorphic euascomycetes also share these characters.

In a study of the phylogeny of species of five ascomycetous teleomorphic yeast genera, Yamada et al. (1998) showed the isolated position of Hyphopichia in partial 18S and 26S rRNA gene sequence-based phylogenetic trees. Therefore, they proposed to accept this genus. This proposal was supported by phylogenetic analyses using D1/D2 sequences (Kurtzman \& Robnett, 1998). Later, accepting Hyphopichia as a genus isolated from other yeast genera, Kurtzman (2005) demonstrated that five Candida species (C. fennica, C. homilentoma, C. rhagii, C. pseudorhagii and C. gotoi) and also Pichia heimii belong to the Hyphopichia clade, consequently introducing the new combination Hyphopichia heimii. Hyphopichia burtonii, the type species of the genus, is a food spoilage organism and was shown to produce styrene that results in off-flavours in fermenting baking

Abbreviation: ITS, internal transcribed spacer.

The GenBank/EMBL/DDBJ accession numbers for the 26S rRNA gene D1/D2 region and ITS region sequences of strain CBS $2455^{\top}$ are GQ389650 and GU246252, respectively.

The Mycobank (http://www.mycobank.org) accession number for $\mathrm{H}$. pseudoburtonii sp. nov. is MB 514824.

The single most parsimonious tree of the ITS sequence alignment is available with the online version of this paper. products with a high sugar content and low water activity (Bonjean \& Guillaume, 2003). It has also been isolated from diverse substrates including various food sources (Kurtzman \& Fell, 1998), freshwater sites and plants such as cacti, and it is also associated with beetles or beetle larval substrates (Ganter, 2006).

In this study, 17 strains previously assigned to H. burtonii, either only on the basis of their molecular characteristics or on the basis of morphological characters, were re-examined phenotypically and phylogenetically. A novel species as well as three distinct groups within the existing Hyphopichia clade are proposed.

The strains studied, their origin and original denomination are listed in Table 1. DNA was extracted from cultures grown on GPYA medium ( $4 \%$ glucose, $0.5 \%$ yeast autolysate, $2 \%$ agar) for three days using the FastDNA kit (Bio 101) with the 'FastPrep' Instrument (Q-Biogene). Primers V9G (de Hoog \& Gerrits van den Ende, 1998) and LR5 (Vilgalys \& Hester, 1990) were used to amplify the partial rRNA gene that includes the D1/D2 domain of the large-subunit rRNA gene as well as the ITS (internal transcribed spacer) domain (ITS1, ITS2 and the intervening 5.8S rRNA) as described by Knutsen et al. (2007). The PCR products were separated by electrophoresis at $80 \mathrm{~V}$ for $40 \mathrm{~min}$ on a $0.8 \%(\mathrm{w} / \mathrm{v})$ agarose gel containing $0.1 \mu \mathrm{g}$ ethidium bromide $\mathrm{ml}^{-1}$ in $1 \times$ TAE buffer $(0.4 \mathrm{M}$ Tris, $0.05 \mathrm{M}$ sodium acetate and $0.01 \mathrm{M}$ EDTA, $\mathrm{pH}$ 7.85) and examined under UV light. The amplicons were sequenced in both directions using the primers LR0R (Vilgalys \& Hester, 1990) and LR5 for the D1/D2 domain, and the primers V9G and ITS4 (White et al., 1990) for the ITS domain. The BigDye Terminator version 3.1 Cycle Sequencing kit (Applied Biosystems) was used according to the manufacturer's recommendations and the products were analysed on an ABI Prism 3730XL DNA Sequencer 
Table 1. Details of strains used for this study

S, Self-sporulating. All strains were originally described as Hyphopichia burtonii, except strains CBS 11416 and CBS 11417.

\begin{tabular}{|c|c|c|c|c|}
\hline Strain & Type strain of: & Origin & $\begin{array}{c}\text { Mating } \\
\text { type }\end{array}$ & $\begin{array}{l}\text { GenBank accession } \\
\text { number for D1/D2 }\end{array}$ \\
\hline \multicolumn{5}{|l|}{ H. burtonii } \\
\hline CBS $2352^{\mathrm{T}}$ NRRL Y-1933 ${ }^{\mathrm{T}}$ & $\begin{array}{l}\text { Pichia burtonii Boidin et al. } \\
\text { Endomycopsis chodati Wickerham }\end{array}$ & Pollen & + & $\mathrm{U} 45712$ \\
\hline CBS 2353 & & Silage, USA & - & GQ389655 \\
\hline CBS $2452^{\mathrm{T}}$ & $\begin{array}{l}\text { Trichosporon behrendi Lodder \& } \\
\text { Kreger-van Rij }\end{array}$ & Sputum, Netherlands & - & GQ389656 \\
\hline CBS 2837 & & China & S & GQ389657 \\
\hline CBS $4571^{\mathrm{T}}$ & Sporotrichum anglicum Castellani & Human, case of chronic bronchitis & - & GQ389658 \\
\hline CBS 5507 & & Bread & + & GQ389659 \\
\hline CBS 5508 & & Diseased caterpillar & - & GQ389660 \\
\hline CBS 5509 & & Skin & - & GQ389661 \\
\hline CBS 6141 & & $\begin{array}{l}\text { Moist barley stored in polythene } \\
\text { bags, UK }\end{array}$ & $S$ & GQ389662 \\
\hline CBS $6375^{\mathrm{T}}$ & Candida fibrae Nakase & Fruit of Musa sapientum, Japan & - & GQ389663 \\
\hline CBS 6559 & & Chicken feed, Guatemala & + & GQ389664 \\
\hline CBS 6560 & & Chicken feed, Guatemala & - & GQ389665 \\
\hline CBS $6874^{\mathrm{T}}$ & Cladosporium fermentans Goto et al. & Oily detritus, Japan & + & GQ389666 \\
\hline CBS $7651^{\mathrm{T}}$ & Trichosporon beijingense $\mathrm{Lu} \& \mathrm{Li}$ & Rotten fruit of Diospyros sp., China & - & GQ389667 \\
\hline \multicolumn{5}{|l|}{ H. pseudoburtonii sp. nov. } \\
\hline CBS $2455^{\mathrm{T}}$ & $\begin{array}{l}\text { Hyphopichia pseudoburtonii } \\
\text { M. Groenewald \& M. Th. Smith }\end{array}$ & $\begin{array}{l}\text { Rumen contents of animal, } \\
\text { Germany }\end{array}$ & + & GQ389650 \\
\hline CBS $5504^{\mathrm{T}}$ & Candida variabilis (Lindner) Berkhout & & - & GQ389651 \\
\hline CBS 5510 & & Bakers yeast, Italy & + & GQ389652 \\
\hline CBS 11416 & & Food, Poland & - & GQ389653 \\
\hline CBS 11417 & & Food, Poland & - & GQ389654 \\
\hline
\end{tabular}

(Perkin-Elmer). A consensus sequence was computed from the forward and reverse sequences with SeqMan from the Lasergene package (DNASTAR). All sequences were assembled and aligned using MAFFT (Katoh et al., 2002) and manual adjustments for improvement were made by eye where necessary using Sequence Alignment Editor (version 2.0a11; Department of Zoology, University of Oxford, Oxford, UK).

The sequence data were analysed by Phylogenetic Analysis Using Parsimony (PAUP) v4.0b10 (Swofford, 2003) and the resulting trees were printed as described by Groenewald et al. (2008). Maximum-parsimony analyses were performed using the heuristic search option with 1000 random taxon additions and the robustness of the trees was evaluated by 1000 bootstrap replications. Other measures calculated included tree length, consistency index, retention index and rescaled consistency index (TL, CI, RI and RC, respectively). Neighbour-joining analyses using different substitution models (HKY85, Kimura two-parameter and uncorrected ' $p$ ') were also executed. In all analyses, gaps were treated as either missing data or fifth character (new state) and large gaps were both included and excluded in different analyses. The sequences were deposited in GenBank (Table 1) and the alignments in TreeBASE (www.treebase.org).
Colony and cell morphology was determined after growth for three days at $25{ }^{\circ} \mathrm{C}$ on GPYA for all strains listed in Table 1. Physiological characteristics were determined by using the API 32C system (bioMérieux) according to the manufacturer's instructions and assimilation of nitrogen compounds and fermentation of glucose were tested using the methods described by Yarrow (1998) for all strains listed in Table 2. Data were retrieved after five days. Growth at $25,30,36$ and $40{ }^{\circ} \mathrm{C}$ was determined by incubation on GPYA for seven days. All tests were replicated.

Ascospore production of all $H$. burtonii strains was reexamined using the tester mating types $+\left(\right.$ CBS $\left.2352^{\mathrm{T}}\right)$ and - (CBS 2353). Five different media were tested, namely 2 and $4 \%$ malt extract agar (Gams et al., 1998), $5 \%$ Difco malt extract, V8 and YM agars (Yarrow, 1998). All cultures were grown alone or mixed with the tester strains. Strains showing no mating reaction with the tester strains were mixed and examined for ascospore production. The cultures were incubated at $25{ }^{\circ} \mathrm{C}$ and inspected at 3-7 day intervals for two months.

\section{Ascospore production}

Of the five media tested, $2 \%$ malt extract agar was found to be the most efficacious for optimal mating. Results of the 
Table 2. Physiological characters of the strains representing Hyphopichia pseudoburtonii sp. nov.

Strains: 1, CBS 2455 ${ }^{\mathrm{T}}$; 2, CBS 5504; 3, CBS 5510; 4, CBS 11416; 5, CBS 11417. All strains were positive for the assimilation of D-galactose, sucrose, $\mathrm{N}$-acetyl-glucosamine, maltose, trehalose, potassium-2-ketogluconate, methyl $\alpha$-D-glucopyranoside, glycerol, palatinose, erythritol, potassium gluconate, D-glucose, glucosamine, L-lysine, ethylamine and cadaverine and fermentation of glucose. All strains were negative for the assimilation of $0.01 \%$ cycloheximide, lactic acid, lactose, inositol, L-rhamnose, melezitose, sodium glucuronate, levulinic acid and nitrate. -, Negative; +, positive; w, weakly positive; $d$, delayed positive.

\begin{tabular}{|c|c|c|c|c|c|}
\hline Characteristic & 1 & 2 & 3 & 4 & 5 \\
\hline \multicolumn{6}{|l|}{ Assimilation of: } \\
\hline L-Arabinose & W & - & - & - & - \\
\hline Cellobiose & + & $\mathrm{W}$ & + & + & + \\
\hline Raffinose & + & w & + & + & + \\
\hline D-Mannitol & + & d & + & + & + \\
\hline D-Sorbitol & + & $\mathrm{d}$ & + & + & + \\
\hline D-Xylose & + & w & + & + & + \\
\hline D-Ribose & W & + & + & + & + \\
\hline Melezitose & + & $\mathrm{w}$ & + & + & + \\
\hline L-Sorbose & W & - & - & W & W \\
\hline
\end{tabular}

mating tests are presented in Table 1. Of the 17 strains identified as $H$. burtonii on the basis of having denticulate conidiogenous cells (Fig. 1a), two isolates were found to be self-sporulating, seven showed mating reactions with the tester strain CBS $2352^{\mathrm{T}}(+)$ and three reacted with tester strain CBS $2353(-)$, all resulting in hat-shaped ascospores. Five strains, CBS 2455 ${ }^{\mathrm{T}}$, CBS 5504, CBS 5510, CBS 11416 and CBS 11417, did not show any mating reaction with both tester strains. However, ascospores were observed in various crossing combinations of these strains. Two strains were of one mating type, designated + , and three isolates were of the opposite mating type, designated - (Table 1). After three days of incubation, conjugation of yeast cells of opposite mating types was observed (Fig. 1b) resulting in asci with 1-4 (mainly 1-2) rough, round to oval ascospores (Fig. 1c-f). These ascospores differ from the observed and reported hat-shaped ascospores of $H$. burtonii and $H$. heimii, respectively.

\section{Physiology}

The physiological characters of CBS $2455^{\mathrm{T}}$, CBS 5504, CBS 5510, CBS 11416 and CBS 11417 are presented in Table 2. The values for existing species did not deviate from published values and are therefore not presented. Growth studies showed that the true $H$. burtonii strains can grow at $36{ }^{\circ} \mathrm{C}$, whereas those similar to strain CBS $2455^{\mathrm{T}}$ are not able to grow at such a high temperature with growth only observed up to $30^{\circ} \mathrm{C}$.

\section{Sequence comparison}

The D1/D2 alignment (deposited in TreeBASE), containing 24 strains including the outgroup sequence, had a total length of 550 characters including alignment gaps, of which 38 were excluded, 266 were constant, 22 were parsimonyuninformative, and 224 were parsimony-informative. Parsimony analysis resulted in two equally most parsimonious trees, one of which is shown in Fig. 2. The two trees obtained differ only with respect to the order of the three species Candida rhagii, C. pseudorhagii and H. heimii. A similar tree topology was found when the gaps were included in the analyses; however, neighbour-joining analyses on this alignment produced different tree topologies (data not shown) and will be discussed below.
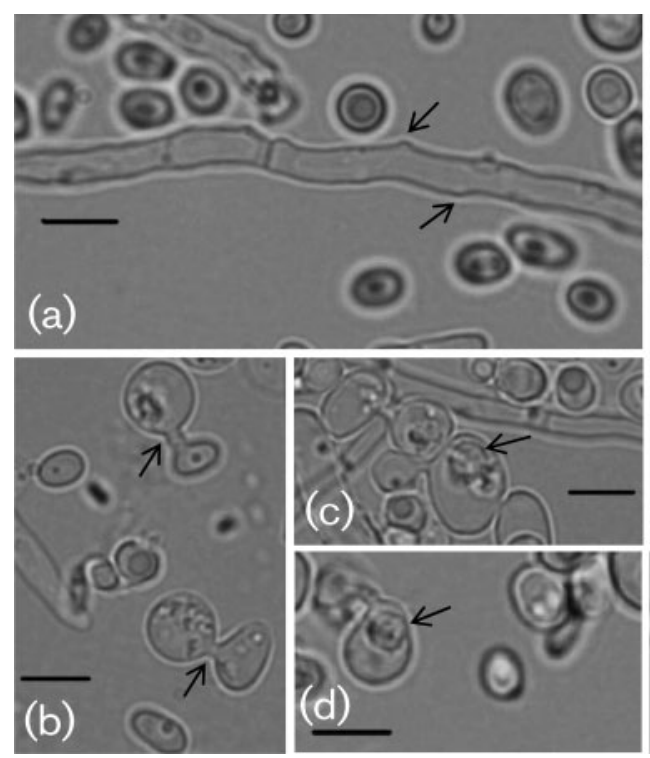
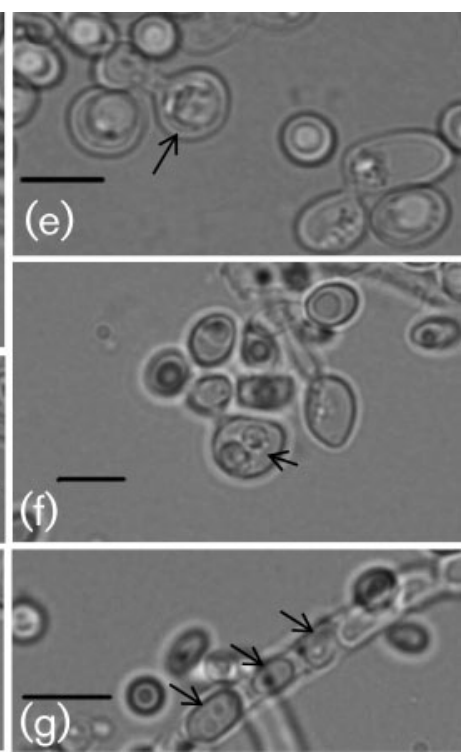

Fig. 1. (a) Denticulate conidiogenous cells of CBS 11416, arrow indicates the scar where conidia were attached; (b) conjugating yeast cells between CBS $2455^{\top}$ and CBS 11416 , arrow indicates conjugation tube; (c-f) asci formed from conjugating yeast cells containing 1-2 spores between CBS $2455^{\top}$ and CBS 11416, arrows show asci with spores; $(\mathrm{g})$ endospores formed by CBS $2455^{\top}$, arrows show endospores. Bars, $5 \mu \mathrm{m}$. 


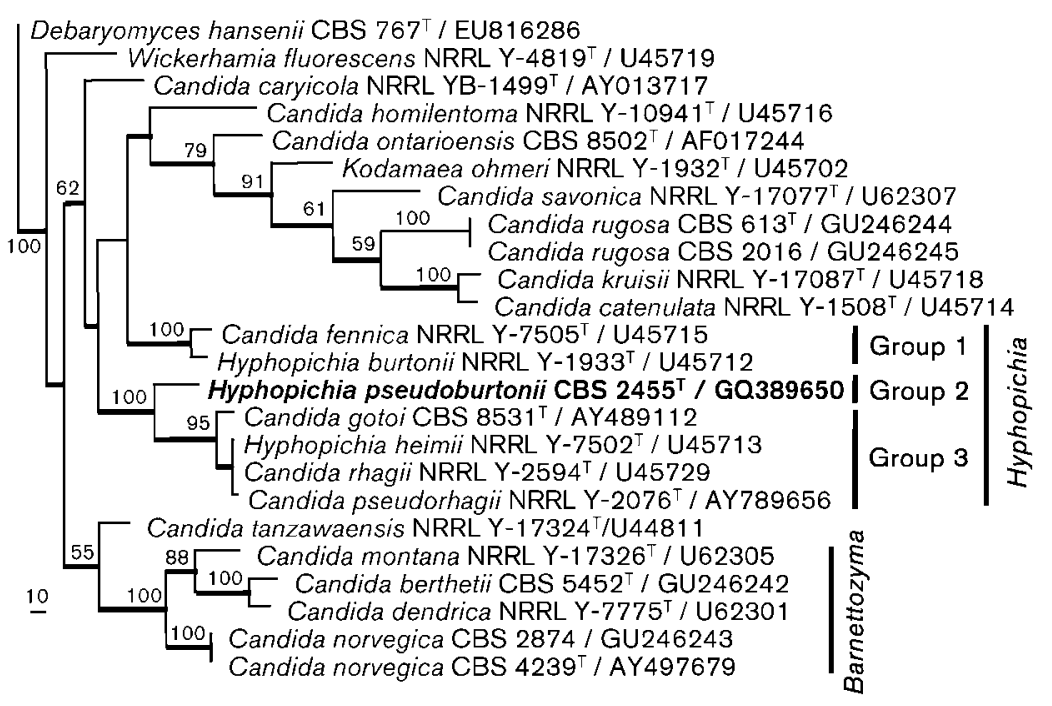

Fig. 2. One of two equally most parsimonious
trees obtained from a heuristic search with
1000 random taxon additions of the $\mathrm{D} 1 / \mathrm{D} 2$
sequence alignment $(\mathrm{TL}=1055$ steps;
$\mathrm{Cl}=0.521 ; \mathrm{Rl}=0.654 ; \mathrm{RC}=0.341)$. Bar, 10
changes per nucleotide position. Bootstrap
support values from 1000 replicates are shown
as percentages at the nodes. Thickened lines
indicate the strict consensus branches. The tree
was rooted to a Debaryomyces hansenii
sequence.

The ITS alignment (deposited in TreeBASE), containing 22 strains including the outgroup sequence, had a total length of 554 characters including alignment gaps, of which 137 were excluded, 166 were constant, 52 were parsimonyuninformative, and 199 were parsimony-informative. Parsimony analysis resulted in a single most parsimonious tree (Supplementary Fig. S1, available in IJSEM Online) that had a similar topology to the trees obtained from the neighbour-joining analyses of the same alignment (data not shown). Previously $H$. burtonii was placed in the Hyphopichia clade together with $H$. heimii, C. fennica, C. rhagii, C. pseudorhagii, C. gotoi and C. homilentoma (Kurtzman, 2005) although there was no significant bootstrap support for this clade in that analysis (less than $50 \%)$. From the data presented in Fig. 2, it is clear that $H$. burtonii, H. heimii, C. fennica, C. rhagii, C. pseudorhagii and $C$. goto $i$ together with the newly described species are still clustering together to some extent. Although $C$. homilentoma clusters together with the species in the Hyphopichia clade using the D1/D2 neighbour-joining analyses (data not shown), all additional analyses done using the D1/D2 and ITS datasets exclude this species from the rest of the Hyphopichia sensu Kurtzman species. In addition, it is difficult to obtain a well-defined clade that contains only the species belonging to Hyphopichia sensu Kurtzman. By using different loci or even different types of analyses for the same locus, additional species that do not belong to Hyphopichia sensu Kurtzman cluster together with these species. The additional species, as well as their positions with respect to the Hyphopichia species within the trees, vary with each parameter change introduced into the analyses. For all of the ITS analyses, C. tanzawaensis and C. caryicola group together with the Hyphopichia species (Fig. $\mathrm{S} 1$ ), whereas for the D1/D2 parsimony analysis, seven Candida species and Kodamaea ohmeri are included (Fig. 2 ). The seven Candida species and Kodamaea ohmeri are split into three groups, one to be found inside $(C$. homilentoma) and two outside the Hyphopichia clade, using the neighbour-joining analysis (data not shown). The dissimilar tree topologies obtained clearly indicate that the Hyphopichia clade is not yet well resolved and analyses using additional loci or sister species (which are not yet known to science) are required. However, the four Candida species that belong to the Barnettozyma clade (Kurtzman et al., 2008) always clustered together, despite the type of analyses done or locus used. This illustrates that the two loci used during this study could be adequately used to place species within a genus that is closely related to Hyphopichia (Kurtzman \& Robnett, 1997). The rearrangement of the species within this Hyphopichia group can also be an indication that more genera are present within this clade. This genus and related species are on long branches at a basal position in the phylogenetic tree of Kurtzman \& Robnett (1997), indicating that these might indeed represent poorly sampled basal lineages. However, due to insufficient data currently available to prove this hypothesis, we propose that three phylogenetic groups should be recognized within the genus Hyphopichia.

Based on the phylogenetic data, strain CBS $2455^{\mathrm{T}}$, previously phenotypically identified as $H$. burtonii, represents a novel species of the genus Hyphopichia. Additional strains, CBS 5504, CBS 5510, CBS 11416 and CBS 11417, were found to have identical D1/D2 sequences to that of strain CBS $2455^{\mathrm{T}}$. The D1/D2 sequences of all the additional $H$. burtonii strains (Table 1 ) were found to be the same as that of CBS $2352^{\mathrm{T}}$. The nucleotide differences found within the D1/D2 domain among strains CBS $2352^{\mathrm{T}}$, CBS $2455^{\mathrm{T}}$ and NRRL Y-7502 ${ }^{\mathrm{T}}$, each representing strains within the three different groups, were 69 alignment positions between CBS $2352^{\mathrm{T}}$ and CBS $2455^{\mathrm{T}}, 83$ between CBS $2352^{\mathrm{T}}$ and NRRL Y-7502 ${ }^{\mathrm{T}}$ and 78 between CBS $2455^{\mathrm{T}}$ and NRRL Y $-7502^{\mathrm{T}}$. The reference area for comparison includes $477 \mathrm{nt}$ ranging from nt 15-492 of the sequence of strain CBS $2352^{\mathrm{T}}$ that is deposited in GenBank as U45712. The nucleotide differences found within the ITS domain among strains CBS $2352^{\mathrm{T}}$, CBS $2455^{\mathrm{T}}$ and NRRL Y-7502 ${ }^{\mathrm{T}}$, 
each representing strains within the three different groups, were 44 alignment positions between CBS $2352^{\mathrm{T}}$ and CBS $2455^{\mathrm{T}}, 52$ between CBS $2352^{\mathrm{T}}$ and NRRL Y-7502 ${ }^{\mathrm{T}}$ and 41 between CBS $2455^{\mathrm{T}}$ and NRRL Y-7502 ${ }^{\mathrm{T}}$. The reference area for comparison includes $402 \mathrm{nt}$ ranging from nt 1-402 of the sequence of strain CBS $2352^{\mathrm{T}}$ that is deposited in GenBank as GU246252.

\section{Taxonomy}

It is evident that the Hyphopichia clade, previously defined by Kurtzman (2005), can be divided into three groups and that Candida homilentoma, once placed in the Hyphopichia clade (Kurtzman, 2005), still has to be linked to an acceptable teleomorphic genus. The Hyphopichia clade is not well supported and, in order to improve the taxonomy of this group, additional loci need to be analysed. Furthermore, the description of additional species belonging to this group will help to strengthen the affinity of the true species of the genus Hyphopichia with each other. In order to unravel the taxonomic relationships among described anamorphic taxa on a broader scale, additional isolates representing teleomorphic species need to be found. This will allow numerous anamorphic yeast species to be connected to teleomorphic genera or species. An additional heterothallic species, represented by strain CBS $2455^{\mathrm{T}}$, was found. Based on the evidence described above, a new species, Hyphopichia pseudoburtonii sp. nov. is described formally below and three groups are recognized within the genus Hyphopichia, with Hyphopichia Group 1 referring to species related to $H$. burtonii, Hyphopichia Group 2 referring to species related to H. pseudoburtonii and Hyphopichia Group 3 referring to species related to $H$. heimii.

\section{Latin diagnosis of Hyphopichia pseudoburtonii M. Groenewald \& M. Th. Smith sp. nov.}

Species heterothallica, status ascigerus Candidae variabilis (Lindner) Berkhout. Post 3 dies $25{ }^{\circ} \mathrm{C}$ in agaro cum dextrose, peptono et extracto levedinis, conidia et cellulae gemmantes ovoidae, elongatae vel cylindricae, $2-4 \times 2.5-6$ (parce -11$)$ $\mu \mathrm{m}$. Culturae albae vel cremae, farinosae, butyrosae, corrugatae, margo integra vel lobata. Pseudohyphae et hyphae septatae. Conidia oriuntur ex hyphae denticulatae. Asci conjugatione duarum cellularum. Ascosporae globosae vel ellipsoidae, (parce) verrucosae. Fermentatio, crescentia in variis substantiis carbonaceus et nitraceis et alii characteres monstrantur in Tabula 2. Holotypus cultura conjugata ascigera CBS $2455^{\mathrm{T}} \times \mathrm{CBS} 5504$, exsiccata et allotypus CBS $2455^{\mathrm{T}}\left(=\mathrm{JCM} 16346^{\mathrm{T}}\right)$ et $\mathrm{CBS} 5504(=\mathrm{JCM} 16347)$ vivus in collectione culturarum Centraalbureau voor Schimmelcultures, Trajectum ad Rhenum praeservantur.

\section{Description of Hyphopichia pseudoburtonii M. Groenewald \& M. Th. Smith sp. nov.}

Hyphopichia pseudoburtonii [pseu.do.bur.to'ni.i. Gr. adj. pseudês false; N.L. gen. n. burtonii a specific epithet; N.L. gen. n. pseudoburtonii the false (Hyphopichia) burtonii (the epithet is chosen because of morphological resemblance to the species Hyphopichia burtonii)].

The species is the heterothallic, ascigerous state of Candida variabilis (Lindner) Berkhout. After 3 days at $25^{\circ} \mathrm{C}$ on glucose-yeast extract-peptone agar, the conidia and yeast cells are oval, elongate to cylindrical and $2-4 \times 2.5-6$ (rarely up to 11) $\mu \mathrm{m}$. The streak culture is white to creamcoloured, farinose, butyrous and wrinkled with an entire or lobate margin. Pseudohyphae and septate hyphae are formed. Conidia from hyphae may arise from denticles. Occasionally endospores are found (Fig. 1g). Asci are formed by conjugating yeast cells. Ascospores are globose to ellipsoidal and rough. Fermentation and growth on various carbon and nitrogen compounds are presented in Table 2.

The holotype of the species, a dried specimen of a sporulating culture of the two mating types CBS $2455^{\mathrm{T}} \times$ CBS 5504, and the allotypes CBS $2455^{\mathrm{T}}$ (=JCM $\left.16346^{\mathrm{T}}\right)$ and CBS 5504 (=JCM 16347), as living strains, are present in the culture collection of the Centraalbureau voor Schimmelcultures, Utrecht, The Netherlands.

\section{Conclusion}

From the phenotypic and phylogenetic studies, the following species with their synonyms are recognized (species listed in alphabetical order).

\section{Hyphopichia burtonii (Boidin et al.) Arx \& van der Walt} (1976)

Anamorph: Candida fibrae Nakase (1971).

Synonyms: Pichia burtonii Boidin et al. (1964); Endomycopsis burtonii (Boidin et al.) Kreger-van Rij (1970); Dematium chodati Nechitsch (1904); Cladosporium chodati (Nechitsch) Saccardo \& D. Saccardo (1906); Candida chodati (Nechitsch) Berkhout (1923); Endomycopsis chodati Wickerham \& Burton (1952) nom. nud.; Sporotrichum carougeaui Langeron (1922); Sporotrichum anglicum Castellani (1937); Trichosporon behrendii Lodder \& Kreger-van Rij (1952); Fermentotrichon behrendii (Lodder \& Kreger-van Rij) Novák \& Zsolt (1961); Candida armeniaca-cornusmas Saruchanyan (1957); Cladosporium fermentans S. Goto et al. (1975); Trichosporon beijingense $\mathrm{Lu} \& \mathrm{Li}$ (1991).

\section{Hyphopichia heimii (Pignal) Kurtzman (2005)}

Synonym: Pichia heimii Pignal (1970).

Hyphopichia pseudoburtonii M. Groenewald \& M. Th. Smith

Anamorph: Candida variabilis (Lindner) Berkhout (1923).

Synonyms: Monilia variabilis Lindner (1898); Oospora variabilis (Lindner) Lindau (1907); Trichosporon variabile (Lindner) Delitsch (1943). 


\section{Acknowledgements}

Wendy Epping is thanked for technical assistance and David Yarrow and Ewald (J.Z.) Groenewald for valuable discussions.

\section{References}

Arx, J. A. \& van der Walt, J. P. (1976). The ascigerous state of Candida chodatii. Antonie van Leeuwenhoek 42, 309-314.

Bonjean, B. \& Guillaume, L.-D. (2003). Yeast in bread and baking products. In Yeasts in Food, Beneficial and Detrimental Aspects, pp. 289-307. Edited by T. Boekhout \& V. Robert. Hamburg: Behr's.

de Hoog, G. S. \& Gerrits van den Ende, A. H. G. (1998). Molecular diagnostics of clinical strains of filamentous basidiomycetes. Mycoses 41, 183-189.

Gams, W., Hoekstra, E. S. \& Aptroot, A. (editors) (1998). CBS Course of Mycology, 4th edn. Baarn: Centraalbureau voor Schimmelcultures.

Ganter, P. F. (2006). Yeast and invertebrate associations. In The Yeast Handbook, Biodiversity and Ecophysiology of Yeasts, p. 303-370. Edited by C. A. Rosa \& G. Péter. Heidelberg: Springer.

Groenewald, M., Daniel, H.-M., Robert, V., Poot, G. A. \& Smith, M. Th. (2008). Polyphasic re-examination of Debaryomyces hansenii strains and re-instatement of D. hansenii, D. fabryi and D. subglobosus. Persoonia 21, 17-27.

Katoh, K., Misawa, K., Kuma, K. \& Miyata, T. (2002). MAFFT: a novel method for rapid multiple sequence alignment based on fast Fourier transform (describes the FFT-NS-1, FFT-NS-2 and FFT-NS-i strategies). Nucleic Acids Res 30, 3059-3066.

Knutsen, A. K., Robert, V., Poot, G. A., Epping, W., Figge, M., HolstJensen, A., Skaar, I. \& Smith, M. Th. (2007). Polyphasic reexamination of Yarrowia lipolytica strains and the description of three novel Candida species: Candida osloensis sp. nov., Candida alimentaria sp. nov. and Candida hollandica sp. nov. Int J Syst Evol Microbiol 57, 2426-2435.
Kurtzman, C. P. (2005). New species and a new combination in the Hyphopichia and Yarrowia yeast clades. Antonie van Leeuwenhoek 88, 121-130.

Kurtzman, C. P. \& Fell, J. W. (1998). Summary of species characteristics. In The Yeasts, a Taxonomic Study, 4th edn, pp. 915947. Edited by C. P. Kurtzman \& J. W. Fell. Amsterdam: Elsevier.

Kurtzman, C. P. \& Robnett, C. J. (1997). Identification of clinically important ascomycetous yeasts based on nucleotide divergence in the $5^{\prime}$ end of the large-subunit (26S) ribosomal DNA gene. J Clin Microbiol 35, 1216-1223.

Kurtzman, C. P. \& Robnett, C. J. (1998). Identification and phylogeny of ascomycetous yeasts from analysis of nuclear large subunit (26S) ribosomal DNA partial sequences. Antonie van Leeuwenhoek 73, 331-371.

Kurtzman, C. P., Robnett, C. J. \& Basehoar-Powers, E. (2008). Phylogenetic relationships among species of Pichia, Issatchenkia and Williopsis determined from multigene sequence analysis, and the proposal of Barnettozyma gen. nov., Lindnera gen. nov. and Wickerhamomyces gen. nov. FEMS Yeast Res 8, 939-954.

Swofford, D. L. (2003). PAUP ${ }^{*}$ : phylogenetic analysis using parsimony (*and other methods), version 4. Sunderland, MA: Sinauer Associates.

Vilgalys, R. \& Hester, M. (1990). Rapid genetic identification and mapping of enzymatically amplified ribosomal DNA from several Cryptococcus species. J Bacteriol 172, 4238-4246.

White, T. J., Bruns, T., Lee, S. \& Taylor, J. (1990). Amplification and direct sequencing of fungal ribosomal RNA genes for phylogenetics. In PCR Protocols - a Guide to Methods and Applications, pp. 315-322. Edited by M. A. Innis, D. H. Gelfand, J. J. Sninsky \& T. J. White. San Diego, CA: Academic Press.

Yamada, Y., Higashi, T. \& Mikata, K. (1998). The phylogeny of species of the ascogenous teleomorphic yeast genera Ambrosiozyma, Hormoascus, Hyphopichia, Arthroascus, and Botryoascus based on the partial sequences of $18 \mathrm{~S}$ and $26 \mathrm{~S}$ ribosomal RNAs. Bull Fac Agric Shizuaka Univ 48, 1-13.

Yarrow, D. (1998). Methods for the isolation, maintenance and identification of yeasts. In The yeasts: a Taxonomic Study, 4th edn, pp. 77-100. Edited by C. P. Kurtzman \& J. W. Fell. Amsterdam: Elsevier. 